\title{
A PROPOSAL FOR A MULTI-DRIVE HETEROGENEOUS MODULAR PIPE-INSPECTION MICRO-ROBOT
}

\author{
ALBERTO BRUNETE, JOSE EMILIO TORRES, MIGUEL HERNANDO \\ and ERNESTO GAMBAO \\ Division de Ingenieria de Sistemas y Automatica, ETSII \\ Universidad Politecnica de Madrid, \\ Jose Gutierrez Abascal 2, Madrid 28006, Spain.
}

\begin{abstract}
This paper presents the architecture used to develop a micro-robot for narrow pipes inspection. Both the electromechanical design and the control scheme will be described. In pipe environments it is very useful to have a method to retrieve information of the state of the inside part of the pipes in order to detect damages, breaks and holes. Due to the different types of pipes that exists, a modular approach with different types of modules has been chosen in order to be able to adapt to the shape of the pipe and to chose the most appropriate gait. The micro-robot has been designed for narrow pipes, a field in which there are not many prototypes. The robot incorporates a camera module for visual inspection and several drive modules for locomotion and turn (helicoidal, inchworm, two degrees of freedom rotation). The control scheme is based on semi-distributed behavior control and is also described. A simulation environment is also presented for prototypes testing.
\end{abstract}

Keywords: Modular, multiconfigurable, heterogeneous, pipe-inspection, behavior-based.

\section{Introduction}

There are many different types of pipes that need to be inspected for several reasons: obstructions, breakages, leakages...etc. Pipes in heating, water and gas systems, placed at home, in buildings or installations (like swimming pools, tanks...etc), are not usually accessible because they are either hidden or cannot be dismantled for inspection. It is difficult to develop a single robot able to explore all of these pipes. In addition, some of these pipes are quite narrow, and most of the commercial robots can not get into them. Thus, a modular micro-robot composed of different types of modules that can be selected depending on the task and that has a diameter of only $27 \mathrm{~mm}$ would ease this task considerably.

Apart from the reasons mentioned above, there are some other good reasons to use modular robots:

- They provide the system with configurability capabilities, giving rise to multiconfigurable, reconfigurable (metamorphic) and auto-configurable systems.

- Increase fault tolerance: a faulty module does not imply system failure.

- Make system scalable: new modules (even with new functionalities) can be added without need of system reconfiguration. 
Up to now, some modules has been developed so far (fig. 1): helicoidal, support and extension (inchworm), rotation and camera. And some others are under development or test: batteries, a sensor module that include different types of sensors like temperature or humidity, and a module to measure the traveled distance.

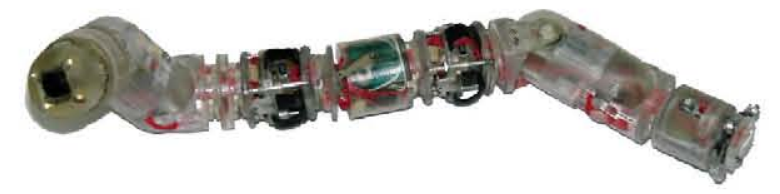

Fig. 1. Micro-robot with the modules: camera/contact, rotation, inchworm and helicoidal

Modular robots can be controlled from different points of view. As opposed to classic model-based AI in the tradition of McCarthy, the reactive-based and behavior-based models in the tradition of Brooks of the "new AI" seems to be a very suitable control scheme for modular field robotics. They present a direct relation with the environment, very important feature in inspection robots that deal with unknown environments and need to react to unpredicted stimuli. Thus, hybrid models that combine aspects of both model-based and reactive-based seems to be the choice of many robots at present time [Kurokawa et al., 2005] [Zhang et al., 2001]. Although some people agree that reactive and behavior-based control is the same, it is not [Mataric, 1992]. Behavior-based control should be considered hybrid, because each behavior can have a different control mechanism, either reactive or deliberate, or even both.

The key part of behavior-based control are behaviors. Generally speaking, a behavior is anything that an organism does involving action and response to stimulation, or the response of a system to its environment. In this sense, Arkin [Arkin, 1998] simply defines it as the reaction to an stimulus. And simpler, anything observable that the robot or system does.

Thus, the actions that a robot performs in each situation can be classified in behaviors. And combining the behaviors in simple situations, the robot can be able to deal with more complicated situations.
Some of the reasons that make behavior based robotics fit in modular field robotics are:

- Connect directly perception (sensors) to action (actuators)

- Allow behaviors to act in parallel

- Allow combination of central control and distributed systems

- Are real-time capable

- Are scalable

In the following sections the development of the electro-mechanical robot and its control will be described in more detail. In section 2 some of the work that has inspired this research is described. On section 3 an overview of the architecture is presented. A mechanical description of the modules used in the research is given on section 4, while the electronic description on section 5 . The simulation environment used for development of prototypes and testing is shown on section 6 . The control architecture is described on section 7 and some tests and results are included on section 8 .

\section{Related work}

Most of the pipe inspection robots nowadays are not modular, i.e. [Roh and Choi, 2004][Klaassen and Paap, 1999]. In order to find related work on modular robots, the best fields are the ones on lattice and chain type modular robots for general purpose.

Even in this field, most of the modular robots that are being developed nowadays are homogeneous (composed of one single type of modules) or composed of two types of modules, one of them passive. Just to cite some of the homogeneous: M-TRAN [Kurokawa et al., 2005], PolyBot [Zhang et al., 2001], CONRO [Shen et al., 2000], Molecube [Zykov et al., 2005], Crystalline [Rus and Vona, 2000], Telecubes [Suh et al., 2002], Superbot [Shen et al., 2006], ATRON [Jorgensen et al., 2004]... etc.

Apart from Tetrobot and CEBOT (well known first designs in this field) and Skyworker, that in some classifications are included as heterogeneous modular robots but its application field has different objectives to those in this ar- 
ticle, the main developments in heterogeneous modular robots are Polypod [Yim, 1994] and ICUBE [Unsal and Khosla, 2000]. There is also a new design in development [Brener et al., 2004].

All of them have only one drive module, being the other one passive. In polypod the passive is a rigid cube shaped module whose main purpose is to hold the batteries and to allow for nonserial chain robots. In I-CUBE is a passive connection element. The difference between these robots and the one we propose is that we deal with different drive modules working together.

A similar research to ours was carried out by David Austin [Jantapremjit and Austin, 2001], who tried to develop a project investigating one form of self-reconfiguring robots that can assemble themselves and reconfigure their hardware to take whatever shape is required for the current task. Unfortunately, due to the difficulty of building this type of mechanism, they had to abandon the project.

Although not heterogeneous, there are some concepts and ideas developed for homogeneous robots that can be broadly used in heterogeneous robots.

It is very interesting the idea of hormones of the CONRO robot [Shen et al., 2000]. A hormone is a signal that triggers different actions in different body parts (modules) and yet leaves the execution and coordination of these actions to local subsystems. A hormone message has a lifetime, has no particular destination but floats in a distributed system and may trigger different actions at different receiving sites. A hormone is terminated when it reaches its destination, when its lifetime expires, or when it has nowhere to go. Since no hormone can live forever, this prevents them from circulating in the network indefinitely. There are three types of hormones: for action specification, synchronization, and dynamic grouping of modules. Since hormones triggers different actions in different modules, it is perfectly applicable to heterogeneous modules.

M-TRAN [Kurokawa et al., 2005] uses a three layer control semi-distributed scheme: is distributed except that there is a master for communication optimization and in autoreconfiguration, that is performed following an event table. The bottom layer contains several functions of the slave controllers directly related to the hardware and an interface between the master and slaves. The middle layer is for communication among modules and realizes mainly two functions; remote control of other modules and a shared memory. In the upper layer, a sequence program designed by the kinematics simulator is interpreted and executed. This three layer scheme is broadly used by many robots and it is also the one we propose. It allows the combination of planning and reactive control layers.

Polybot [Zhang et al., 2001] describes a software architecture that features a multi-master/ multi-slave structure in a multithreaded environment, with three layers of communication protocol. One of its main contributions is the attribute/service model. Attributes are abstractions for shared memory/resources among multiple threads located in one or more processors. E.g. desired joint angle. Services are abstractions of hardware or software routines. This is a very interesting feature because it allows sharing resources amongst modules.

CAMPOUT [Huntsberger et al., 2003] is an architecture for mobile robots, and although it is not designed for modular robots, it defines some types of behaviors that are very interesting and also applicable to modular robots: primitive, composite (combination of primitives), communication, shadow (behaviors that reside in other modules but can be used remotely), cooperation/coordination (allow cooperation between modules).

\section{Architecture overview}

At this point some of the problems that arise from the use of heterogeneous modular robots have been already mentioned, as they are the same as in homogeneous robots:

- Synchronization

- Communication

- Action specification / Control

From the use of several drive modules a new problem appears: the combination of different locomotion modes. This is what really makes heterogeneous modular robots different from the 
homogeneous ones. And it is what this paper tries to deal with.

The architecture presented in this paper tries to set up the basis for the development and control of chain-type modular robots (of very small size) composed of different types of modules and several drive modules (understanding by that modules with locomotion capabilities, see [Brunete et al., 2007]).

The control architecture proposed is based on behaviors (as described in section 1). Each behavior takes care of one feature of the robot. Some of them will manage the hardware acting as reactive components, others will plan the next step acting as deliberative components, some other will be hybrid...etc.

Having different behaviors each of them trying to make the robot do what they want, creates the necessity of coordination between them in order to select the most appropriate or the most relevant action to take when facing a particular situation, what is called the action-selection problem [Pirjanian, 1999].

The coordination mechanisms and the behaviors developed will be described further on. We will discuss first the hardware and electronics in next sections.

Other important point on the architecture, apart from the behavior coordination, is an interpreter between the modules (since they are different) and the higher lever control (or behavior). This will be discussed on section 7 .

\section{Mechanical description}

In this section the different types of modules that are available will be described, as well as the common interface that allows all modules to be connected between them (even some of the previous versions of the modules [Brunete et al., 2005])

\subsection{Interface}

The common interface allows mechanical and electrical connection between modules. The electrical bus is composed of 8 wires:

- Power (5v) and ground

- $I^{2} C$ communication: data and clock
- Two synchronism lines (In and Out)

- Two auxiliary lines.

In the beginning there was only one synchronism line, but for bidirectional communication two are needed. The synchronism line is used for low level communication between adjacent modules. It is a kind of peer to peer communication, unidirectional. The communication along the micro-robot is from module to module, and it seems like passing a token. Thanks to this line, every module can be aware of which other modules are close to him, and the central control of the robot is able to know which is the configuration of the micro-robot. This wires are connected directly from the digital port of one microcontroller to a digital port of the other. One line to read, and one to write, thus bidirectional communication is performed.

The auxiliary lines are for general purposes. They can be used, for example, to carry the video signal from the camera.

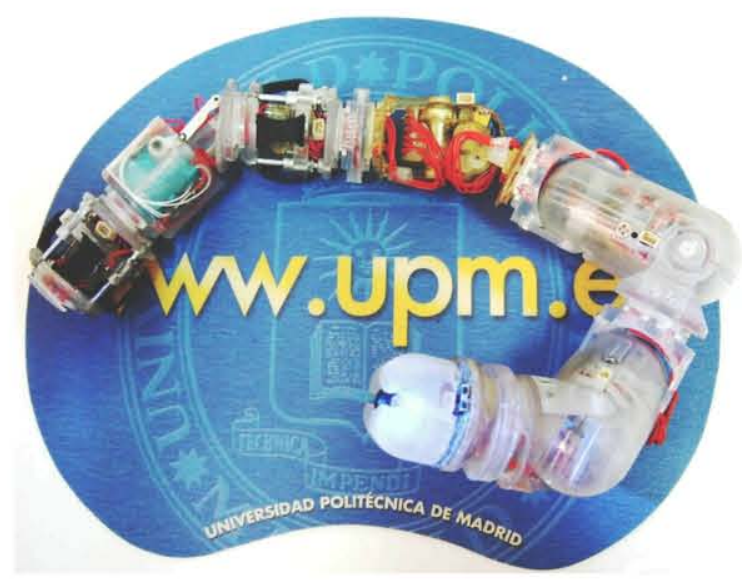

(a)Camera, new rotation(2), old rotation, new support and rotation (inchworm) modules

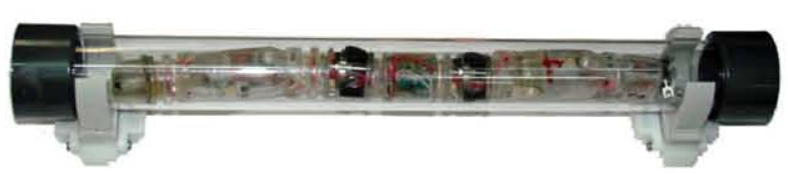

(b) The robot inside a $40 \mathrm{~mm}$ diameter pipe

Fig. 2. Prototypes

\subsection{Real Modules}

The prototypes developed stands out for a new electronic incorporating accelerometers, robust- 
ness and some other features that will be discussed later on. These are: a new rotation module, a new inchworm module (with support and extension modules) and a new camera module (fig. 2). The maximum diameter of all of them is $27 \mathrm{~mm}$. Some part thicknesses are less than $1 \mathrm{~mm}$. Table 1 shows the main characteristics of all of them.

Table 1. Modules characteristics

\begin{tabular}{cccc}
\hline Module & Length $[\mathrm{mm}]$ & Diameter $[\mathrm{mm}]$ & Weight $[\mathrm{g}]$ \\
\hline Camera & 25 & 27 & 6,5 \\
Support & 27 & 27 & 12,5 \\
Extension & 30 & 27 & 16 \\
Rotation & 64 & 27 & 27 \\
Helicoidal & 28 & 27 & 15 \\
\hline
\end{tabular}

\subsubsection{Camera/Contact module}

The camera module (fig. 3) takes a very important part in information acquisition of the environment, in order to detect holes, breakages or cracks in the pipes. The module is provided with a CMOS B\&W camera which allows to visualize the inner part of the pipe and with contact sensors which allow to detect obstacles (i.e. turns) inside the pipe.

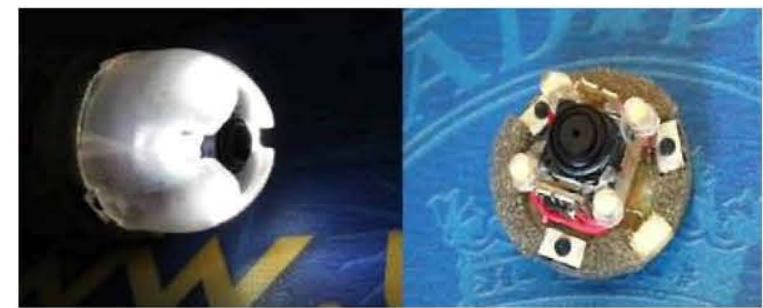

Fig. 3. Camera module (with leds and contact sensors)

The camera is switched on and off through a MOSFET. Additionally, the LEDs for the illumination are also controlled through a MOSFET, letting the microcontroller to vary the intensity of light by generating a PWM sign.

\subsubsection{Rotation module}

The rotation module (fig. 4) is a two degrees of freedom module that allows rotations in the hor- izontal and vertical planes. A set of this modules put together (fig. 5) can perform an ondulatory movement (snake-like) that makes the robot go forward .

In order to make it as robust as possible, a commercial servomotor has been chosen as opposed to previous modules in which a modified gearset was used (the modification made it more compact but there was a lack of torque [Brunete et al., 2005]). It is a CS-101 servomotor with a torque of $0.7 \mathrm{~kg} \cdot \mathrm{cm}$ at $4.8 \mathrm{v}$. The new chassis protects the electronics improving the robustness of the module.

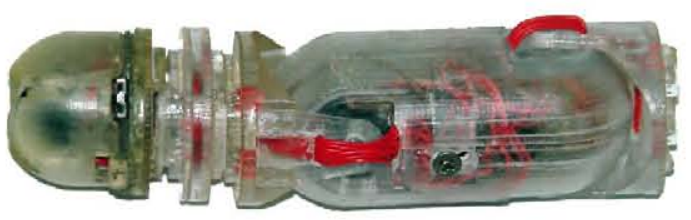

Fig. 4. Rotation module plus camera

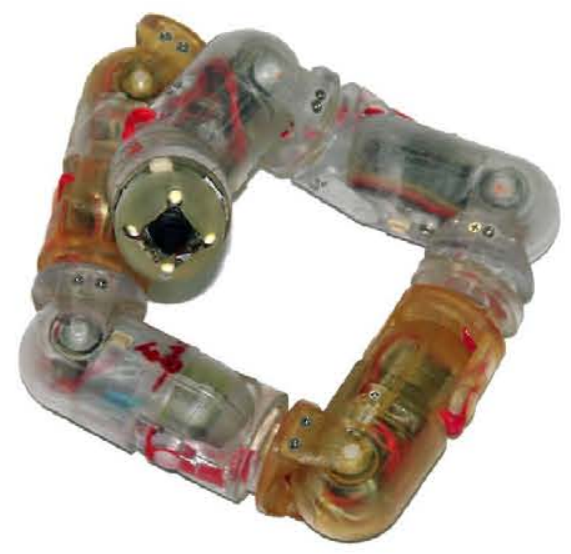

Fig. 5. Snake configuration plus camera

\subsubsection{Inchworm modules}

In order to perform worm-like movements, two modules have been developed: an extension module and a support module (fig. 6). The inchworm's mode of locomotion allows the robot to maneuvering in extremely small spaces. Another advantage of this kind of motion is that the robot manage to maintain a firm grip on the surface at all times while other types of motion, as for example the helicoidal, display a tendency to slip as the slope increases. The support mod- 
ule is used to fix the micro-robot to the pipe, so this module does not move. And the extension module is used to expand the robot (make it go forward), and to turn to right and left.

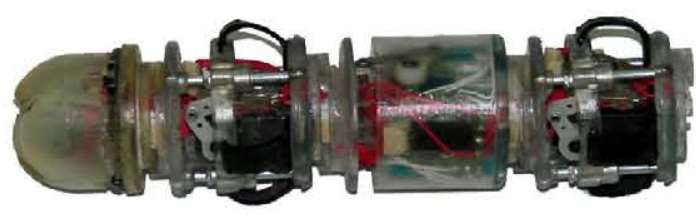

Fig. 6. Inchworm module plus camera

The support module consists in four rubber bands positioned around the module at 90 degrees from each other. In order to expand, the servomotor of the module pushes a ring where all the bands end, and as a result of this movement the bands are bent and fixed to the pipe, so the module gets a grip to the pipe.

The extension module is the new contribution. It is based on a parallel robot with two arms linked to the base and to the final platform. The relative movement of the two arms (each of them driven by a servomotor), change the length of the module and the angle of the second platform. Consequently the module can go forward and also turn.

\subsubsection{Helicoidal module}

The helicoidal module (fig. 7) was designed to be a fast drive module able to push other modules. It is composed of two parts: a body and a rotating head.

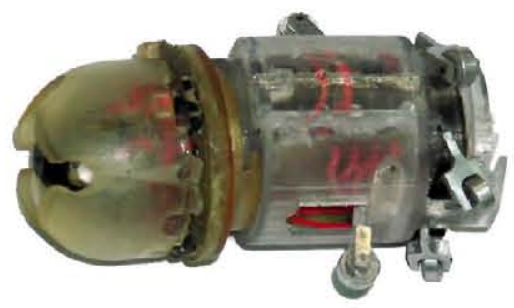

Fig. 7. Helicoidal module plus camera

When the head turns, it goes forward in a helicoidal movement (helped by the distribution of the wheel making a 15 degrees angle with the vertical) that pulls the body of the micro-robot forward. The wheels of the body help to keep the module centered in the pipe.

\subsection{In-process Modules}

There are some other modules that are being designed at the moment. One of them is being tested in the simulator before it is built up. It is a module to compute the traveled distance by using several wheels provided with encoders and the development of an algorithm.

Another one is a sensory module that include different types of sensors, like temperature, humidity...etc.

Finally, a battery module has been already developed but with no good results, so it is being improved in order to give the necessary power supply to drive at least some of the modules for a reasonable period of time.

\subsection{Possible Configurations}

There are several configurations in which the modules can be assembled. Some of them that has been already tested in the simulator are (fig. 8):

- a snake-like robot + camera

- a worm-like module composed by support and extension modules (Config. 1)

- a worm-like + rotation + camera (Config. 2)

- a helicoidal drive module (+support) (+rotation) + camera (Config. 3)

- a several support + several rotation (Config. 4)

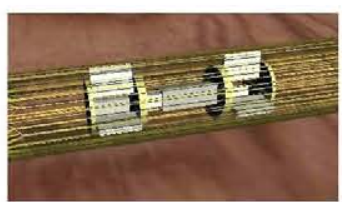

(a)Config. 1

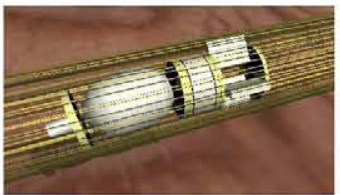

(c)Config. 3

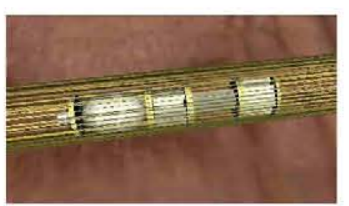

(b)Config. 2

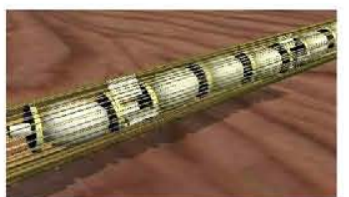

(d)Config. 4
Fig. 8. Possible configurations 


\section{Embedded electronics description}

Each module includes an electronic control board which performs the following tasks:

- Control of actuators

- Communication via I2C, or with adjacent modules

- Manage several types of sensors

- Auto protection and adaptable motion

- Self orientation detection

- Low-level embedded control

The low-level control will be described in section 7 . The remaining two most interesting features, the auto protection and adaptable motion and the self orientation detection will be described next.

\subsection{Auto protection and adaptable motion}

The auto-protection control is based in the control scheme shown in fig. 9.

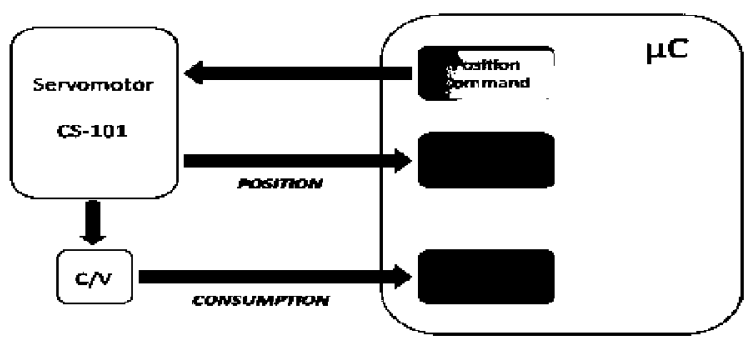

Fig. 9. Auto-protection control scheme

Actuators control is based in two feedback loops, position and consumption. This allows the module to prevent harms to its servomotors if they try to reach an impossible position, for example due to obstacles. Additionally, thanks to these feedback loops, it is possible to implement a torque regulation to avoid high consumption when it is no needed. That is very useful, since the modules require more energy when climbing a vertical pipe than when moving in horizontal.

If by any cause a servomotor gets stuck, the consumption remains at his top value for a long period, as it is shown in fig. 10, as opposed to fig. 11 that shows a normal output. Thus, it is easy to detect these problems and cut power to the servomotor to avoid its damage.
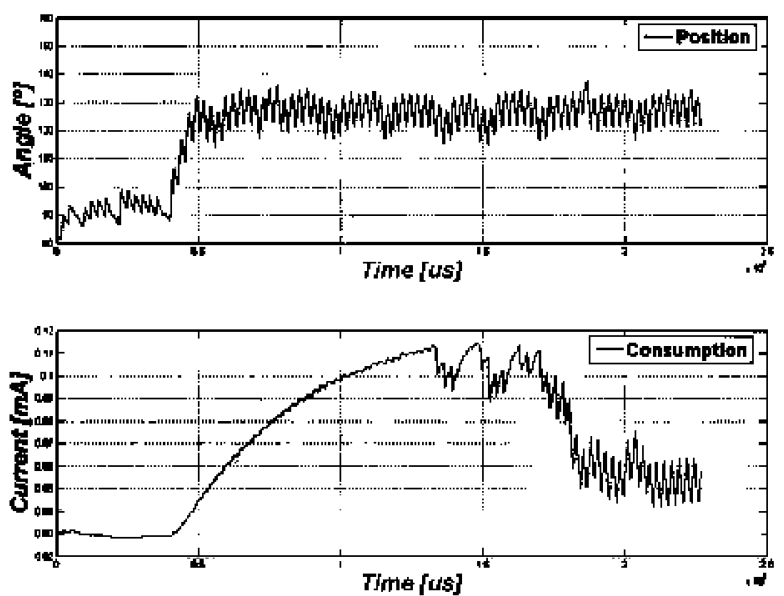

Fig. 10. Consumption output when servo blocking
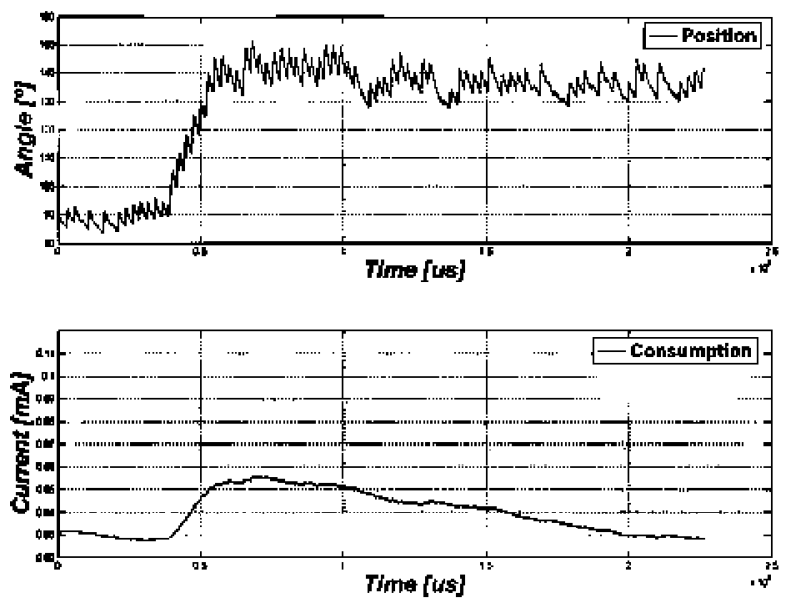

Fig. 11. Normal consumption output (non blocking)

\subsection{Self orientation detection}

New modules are equipped with three-axis accelerometers. With these new sensors inside the micro-robot, it is possible to know how the robot is orientated respect the ground, measuring the acceleration vector of gravity, and towards direction it is moving.

The three-axis accelerometer used is the MXR9150. This sensor can measure $\pm 5 g$ with a sensitivity of $150 \mathrm{mV} / \mathrm{g}$ at $3.0 \mathrm{~V}$. And it's able to detect both dynamic (e.g. movement) and static acceleration (e.g. gravity). The MXR9150 provides three radiometric analog outputs that are 


\section{Embedded electronics description}

Each module includes an electronic control board which performs the following tasks:

- Control of actuators

- Communication via $\mathrm{I} 2 \mathrm{C}$, or with adjacent modules

- Manage several types of sensors

- Auto protection and adaptable motion

- Self orientation detection

- Low-level embedded control

The low-level control will be described in section 7 . The remaining two most interesting features, the auto protection and adaptable motion and the self orientation detection will be described next.

\subsection{Auto protection and adaptable motion}

The auto-protection control is based in the control scheme shown in fig. 9 .

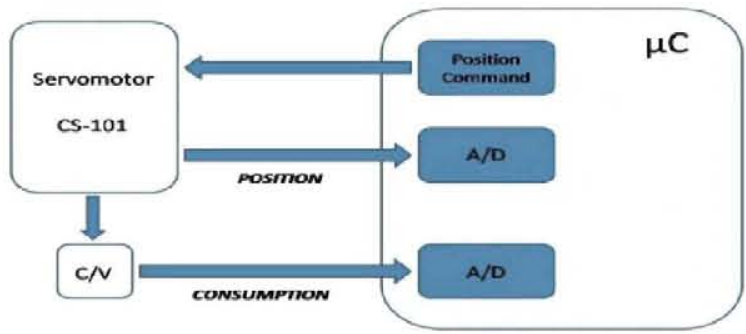

Fig. 9. Auto-protection control scheme

Actuators control is based in two feedback loops, position and consumption. This allows the module to prevent harms to its servomotors if they try to reach an impossible position, for example due to obstacles. Additionally, thanks to these feedback loops, it is possible to implement a torque regulation to avoid high consumption when it is no needed. That is very useful, since the modules require more energy when climbing a vertical pipe than when moving in horizontal.

If by any cause a servomotor gets stuck, the consumption remains at his top value for a long period, as it is shown in fig. 10, as opposed to fig. 11 that shows a normal output. Thus, it is easy to detect these problems and cut power to the servomotor to avoid its damage.
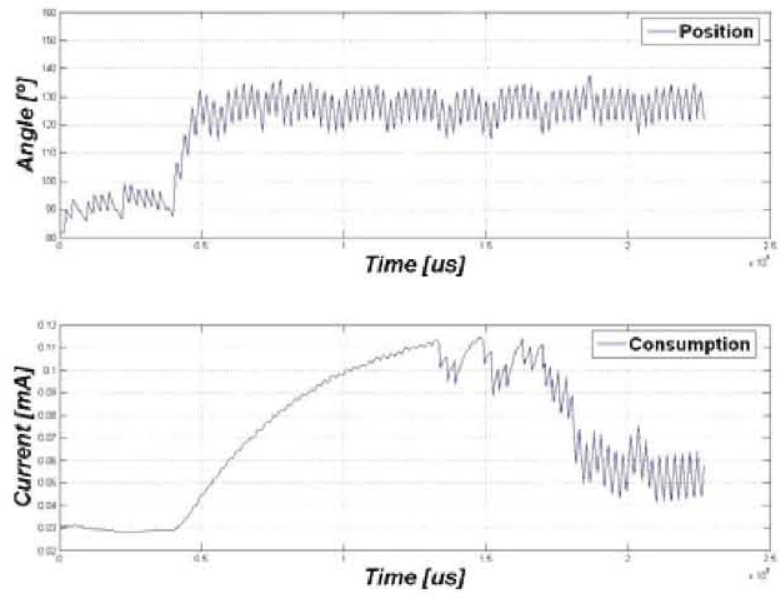

Fig. 10. Consumption output when servo blocking
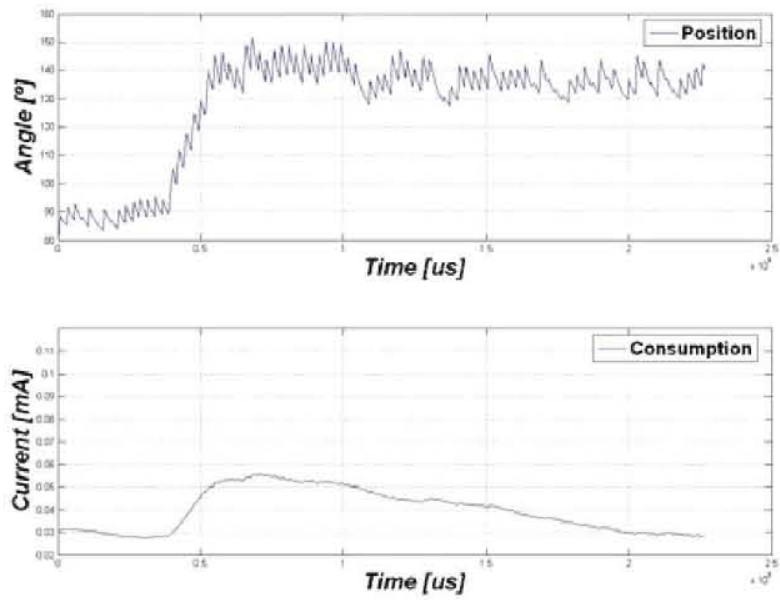

Fig. 11. Normal consumption output (non blocking)

\subsection{Self orientation detection}

New modules are equipped with three-axis accelerometers. With these new sensors inside the micro-robot, it is possible to know how the robot is orientated respect the ground, measuring the acceleration vector of gravity, and towards direction it is moving.

The three-axis accelerometer used is the MXR9150. This sensor can measure $\pm 5 g$ with a sensitivity of $150 \mathrm{mV} / \mathrm{g}$ at $3.0 \mathrm{~V}$. And it's able to detect both dynamic (e.g. movement) and static acceleration (e.g. gravity). The MXR9150 provides three radiometric analog outputs that are 
set to $50 \%$ of the power supply voltage at $0 \mathrm{~g}$, $1.5 \mathrm{~V}$ in our case.

The following figures show the results of some experiments. Figure 12 shows the output of the accelerometers when the module is moving along a linear trajectory in the XY plane, forward and backwards. The signals are very clear. In the $\mathrm{Z}$ axis there is no variation while in the $\mathrm{X}$ and $\mathrm{Y}$ axis the signals rise and fall when the module moves forward and backward.

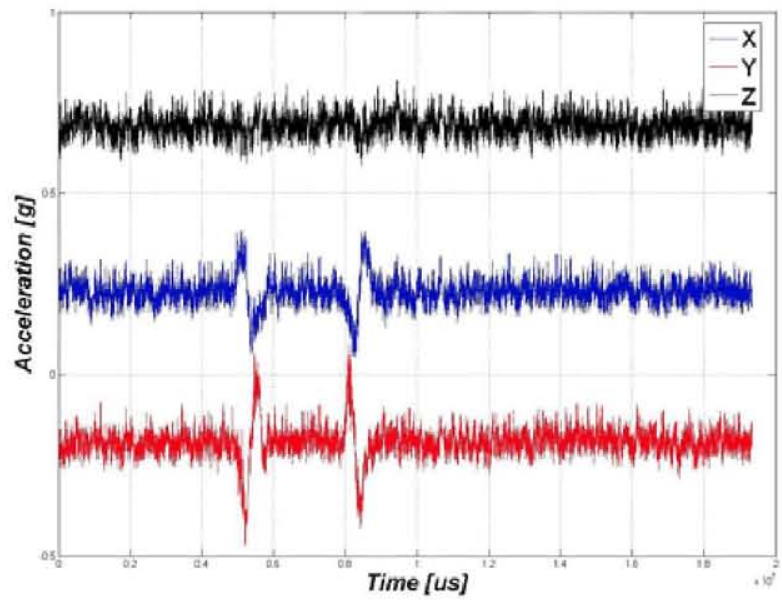

Fig. 12. Module moving along a linear trajectory in the $\mathrm{XY}$ plane
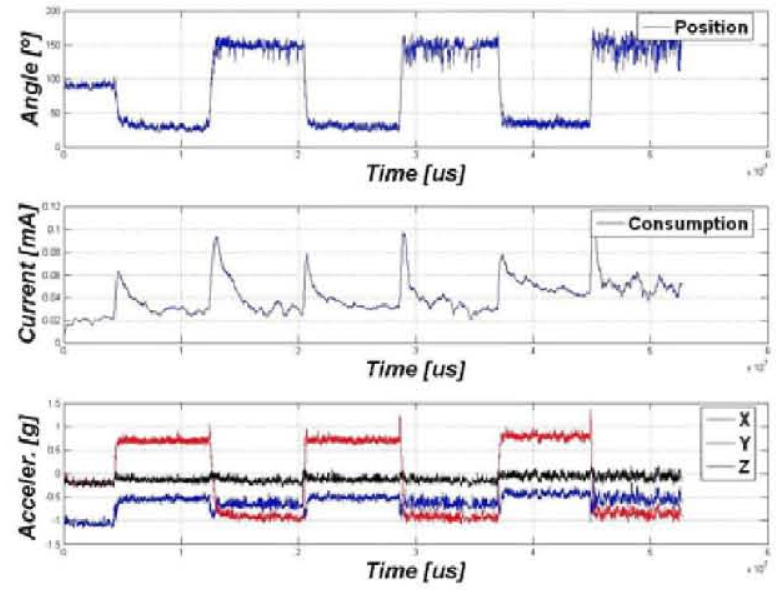

Fig. 13. Servo moving from $30^{\circ}$ to $150^{\circ}$ with no load

In figure 13 the rotation module is moving one servomotor from 30 to $150^{\circ}$ with no load (about $0,35 \mathrm{~kg} * \mathrm{~cm}$ ). The top figure shows the rotated angle. In the middle figure, it is possible to see that the consumption increases (peaks) every time the servo moves, but is stable when the servo is still. In the bottom figure the output of each axis of the accelerometer is drawn, showing a transition (peak up or down) every time the servo moves. Thus the direction of movement can be computed.

Figure 14 shows the results obtained moving from 150 to 30 degrees but with a total load of $0,81 \mathrm{Kg}^{*} \mathrm{~cm}$. Apart of some stabilization problems in the beginning it can be observed that the results are similar to the test without load.

The incorporation of the accelerometers allows also to recognize different patterns of locomotion of each type of module. For example, in figure 15 a pattern of the movement of the inchworm modules can be generated from the data obtained from the accelerometers.
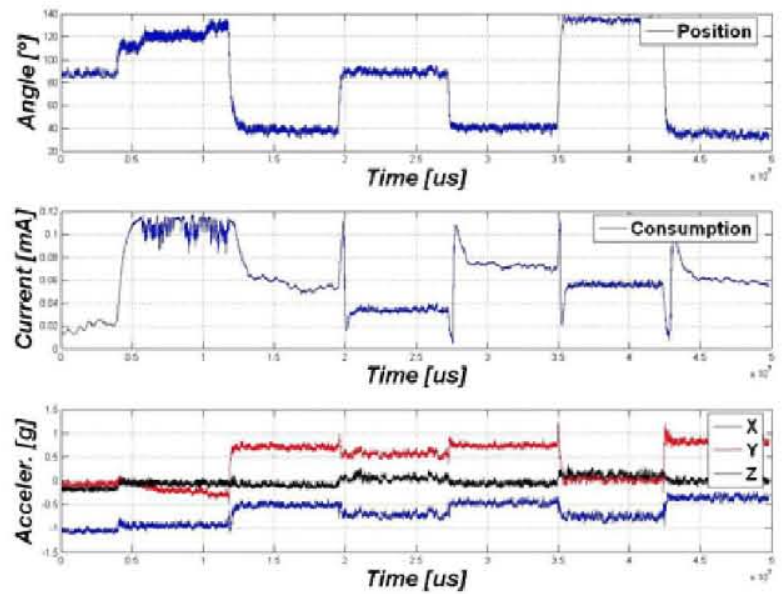

Fig. 14. Servo moving from $150^{\circ}$ to $30^{\circ}$ loaded

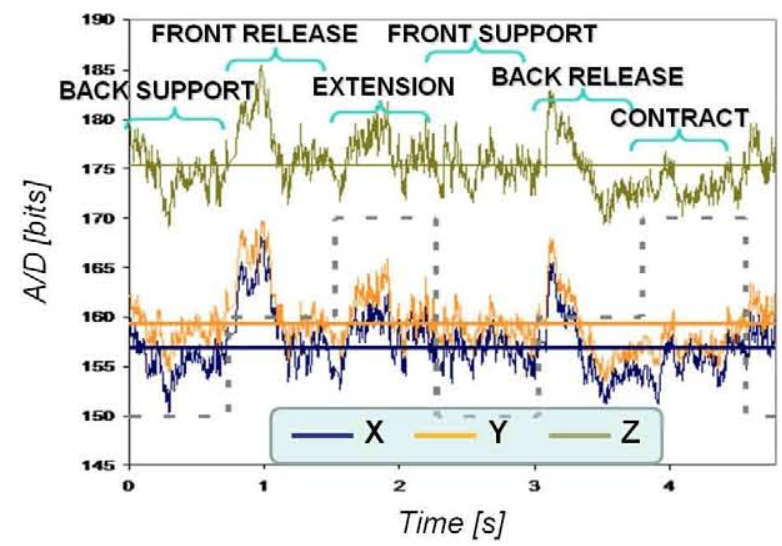

Fig. 15. Inchworm motion pattern 


\section{Simulation environment}

A simulation environment has been developed to provide an efficient way to test prototypes and verify control algorithms, hardware design, and exploring system deployment scenarios.

It can also be used to verify the feasibility of system behaviors using realistic morphology, body mass and torque specifications for servos.

The simulator (fig. 16) is built upon an existing open source implementation of rigid body dynamics, the Open Dynamics Engine (ODE), an open-source physics simulation API, which allows to perform on line simulation of rigid body dynamics, and to define wide variety of experimental environments and actuated models.

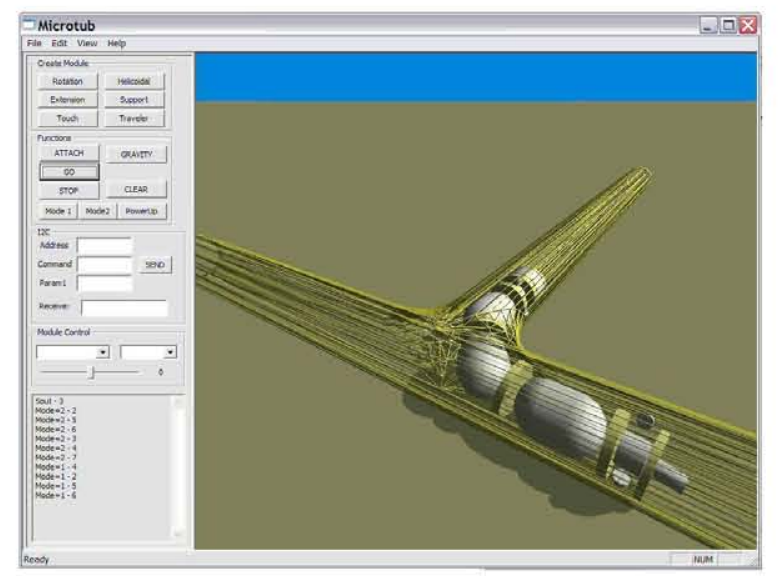

Fig. 16. Simulation Environment

Although ODE has some primitives to simulate motors, in order to make a simulation closer to reality a servo model and a new class has been designed.

For the purposes of better performance and stability, the model was simplified to a set of standard geometrical primitives (such as spheres, cylinders, capsules and cubes).

The created geometric morphology model was assigned dynamic properties that correspond to estimated specifications. E.g. Masses. Degrees of freedom were limited by the maximum torque and speed of the servomotors. Friction coefficients were set to values estimated for materials to be used for module manufacturing and possible surface materials.

In order to emulate the control program in each of the modules a thread is created for each of them. This thread is equivalent to the program that is running inside of each microcontroller on the modules. Thus, the time of execution of both routines, should be equivalent. The communication between adjacent modules through the synchronism line and the $I^{2} C$ communication are also simulated, trying to keep the highest fidelity between reality and simulation.

\section{Control scheme}

The control architecture that is going to be described is aimed to modular robots composed of different types of modules (heterogeneous modules) that can be arranged in different configurations, what is called multiconfigurability. Thus, the robot can be manually assembled in different configurations depending on the chosen task.

The different modules of the robot have to be manually assembled at the beginning. But it is not desired to reprogram each module every time a new configuration or a new task is chosen. Here is where the control architecture comes in. Thanks to this control architecture, the robot is able to receive simple orders and execute them no matter the configuration it has. Example: go, stop, turn, explore...etc.

\subsection{Description}

At this point the architecture has been designed to have a control planner that takes decisions for the whole robot. But also, the robot, and indeed every module, has to be capable to react in real time to unpredicted events. The control is divided into a central control (CC) and an onboard control.

- Central Control (CC): It could be a PC or one of the modules. Nowadays it is a PC. In the future it will be one of the modules in order to make the robot autonomous. It sends commands to each module to tell them the behavior to follow.

- Onboard Control: it is embedded in each module and it is based on behaviors. It allows the modules to react in real time (i.e. to 
sense external and internal stimuli, as overheating, unreachable positions, adapt to the pipe shape...etc), to perform tasks that don't need the $\mathrm{CC}$ (to communicate with adjacent modules, move...etc).

One of the behaviors onboard the modules will act as an interpreter between the central control and the onboard control, the heterogeneous behavior. The heterogeneous behaviors of all modules form the heterogeneous layer, one of the main features of this paper. It is called a middle layer because it acts between the $\mathrm{CC}$ (highest level layer) and the onboard control (a kind of low level layer although it is hybrid as well).

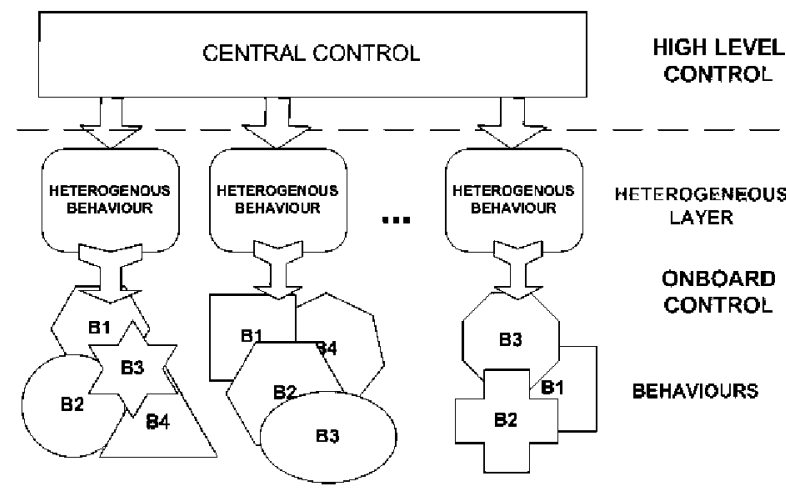

Fig. 17. Control Layers

Thus, the control can be divided into three layers(fig. 17):

- Low Layer: Based on behaviors. Controls the movements of the module and the reaction to unexpected external stimuli. Protects against motor overheating while trying to get to unreachable positions.

- Heterogeneous (Middle) Layer: behavior that translates commands coming from the $\mathrm{CC}$ into specific module commands.

- High Control Layer: Control the robot as a whole. May specify the behavior at each instant and act as an arbiter. It is in charge of planning.

The robot is controlled as a whole, taking into account the current configurations of the robots (all modules). There is no need to send specific commands to each module every time (only when an specific order is to be send to a specific module, for example to retrieve data from it). Every module can performs individual actions and behave in different way to the same commands.

The modules plus the $\mathrm{CC}$ can behave autonomously (from the point of view of control, it still needs to be power supplied), without any need of human intervention.

\subsection{Defined Behaviors}

There are two types of behaviors: survival behaviors and target behaviors. Survival behaviors have the highest priority and its purpose is to keep the robot alive: avoid overheating, avoid damage to the actuators, avoid mechanical damages...etc. The target behaviors are aimed to achieve the goal of the mission and have equal priorities.

The behaviors that have been defined are:

(i) Target behaviors:

(a) Star-tup: in order to know the initial configuration.

(b) Exploration - Go forward/backwards (that includes elbow and bifurcation negotiation,obstacles...etc).

(c) Reach a target

(d) Stop / Stand-by mode

(e) Self-verification (to check if all modules are working properly)

(f) Retrieve data from the environment: image, temperature, humidity...etc.

(ii) Survival behaviors:

(a) Avoid overheating

(b) Avoid damage to the actuators

(c) Avoid mechanical damages

Each module knows what actions it has to perform for every behavior depending on the situation and state it is in every moment. However, this actions can be modified by the reactive layer (survival behaviors) in case of emergency.

For target behaviors, it is the $\mathrm{CC}$ the one that decides which one has to be executed in every moment and when to change the behavior. 


\subsection{Behavior coordination}

There are two levels of coordination: Between the target behaviors the coordination mechanism selected is cooperative. All modules have to cooperate to achieve the goal. The survival behaviors have the highest priorities, so here the coordination mechanism is competition. The one with the maximum urgency takes the control and subsumes the target behaviors.

\subsection{Behaviors}

A description of two implemented behaviors is given next:

\subsubsection{Start-up}

After mechanical connection of modules and power up, the phase of awareness starts: every module get to know its position in the modular chain. This is done with the following procedure:

The CC sends a $\left(I^{2} C\right)$ message to all the modules. All modules activate its synchronism line. The one who is the first one replies (it knows that it is the first because the synchronism line is down). The CC sends a $\left(I^{2} C\right)$ message to all the modules. The first module put the synchronism line down. The second module replies. And so on.

After that the robots remains in a stand by phase waiting for an objective. When an objective is selected (i.e. "go"), the CC sends an $I^{2} \mathrm{C}$ message to every module with the behavior to follow. It is important to remark that all messages are the same, no matter which module they are aimed to. The heterogeneous layer of each module translate this message to proper commands of the module.

Every certain time, the CC sends a message to all the modules demanding if they have something to say (polling). That is the way in which the modules can communicate with the $\mathrm{CC}$ or with other modules.

\subsubsection{Exploration}

In a straight pipe the movement of the robot is quite systematic, and it depends mainly, of which types of modules the robot is composed of, and what are the predominant modules.
If the predominant modules are the rotation modules, a snake-like gait is performed. There is a lot of bibliography about snake-like movements [Gonzalez et al., 2006]. The snake-like movement is achieved by means of a typical sinusoidal wave propagating through the modules (Eq. (1))

$$
P o s=A * \sin (\omega * t+\phi)
$$

A, $\omega$ and $\phi$ are parameters of the modules, and " $t$ " is a counter that is increasing periodically. " $\mathrm{t}$ " is reseted at the same time for all modules when a start sequence message is received from the $\mathrm{CC}$. This is the way modules synchronized.

If the predominant modules are the support and the extension modules, an inchworm gait is performed. In order to perform a worm-like movement, the support and extension modules have to be connected together (support + extension + support). The sequence of movement is as follows:

(i) The rear module expands (making pressure against the pipe) and the front one releases.

(ii) The central module expands straight or in angle.

(iii) The front module expands and the rear one releases.

(iv) The central module contracts.

As well as the rotation module, these modules have a counter that shows them when they have to perform each step. When they received the command to move they reset the counter in order to be synchronized.

The helicoidal module has only one degree of freedom. It is able to go forward or backwards pushing other modules. Thus, an helicoidal module can be added to the other modules and its push will be added to the other modules push.

Other modules that have no actuators act as pig modules, they are carried out by the drive modules. They only have to pass the signals coming from the synchronism line.

The procedure changes when there are bifurcations. Here the touch (and camera) module plays a very important role because it is the one that has touch sensors to detect obstacles, in this case elbows and bifurcations. When the 
touch module detects an obstacle sends a message to the $\mathrm{CC}$, and the $\mathrm{CC}$ sends a message to all the modules asking them to change to the right behavior.

When the robot detects and elbow or bifurcation, the CC sends a message to every module pointing out that the modules have to pass to the behavior "elbow". The sequence is as follows (fig. 18):

- The first module (M1) turns 90 degrees.

- M1 turns up the synchronism line with module M2.

- When M2 detects the Sinc. line up, M2 turns 90 degrees.

- When M2 has turn a predetermined angle (about 60 degrees), M2 turns downs the Sinc. line with M1.

- M1 gets back to the initial 0 degrees position.

- When M2 has turned 90 degrees turns up the Sinc. line with M3 and so on... etc

Passive modules have nothing to do but to pass the token to the next module (through the synchronism line).

This algorithm allows the robot to go forward by pushing against the pipe walls. It is an innovative solution because it includes $\mathrm{P} 2 \mathrm{P}$ communication between adjacent modules besides the $I^{2} C$ communication. An slave module can only communicate with adjacent modules. Since the robot is a chain-type robot, this is considered sufficient for the purposes of this architecture.

\section{Tests and results}

Several tests have been performed to prove each type of locomotion: helicoidal, inchworm (two support modules plus one extension module) and snake-like. Table 2 shows the speed of the robot at different angles and with different configurations.

The main control tests have been carried out in the simulator. Several configurations (including the ones mentioned in section 4.4) have been successfully tested. As an example, in figure 18 an elbow negotiation performed by rotation and camera / contact modules can be seen. In this example the micro-robot receives the command "go" and starts going forward. When it detects an obstacle (thanks to the contact sensor) it autonomously turns and negotiates the elbow.

Table 2. Speed and slope for different configurations

\begin{tabular}{ccc}
\hline Slope & Speed $[\mathrm{cm} / \mathrm{s}]$ & Modules involved \\
\hline $0^{\circ}$ & 2,5 & Inchworm \\
$30^{\circ}$ & 2 & Inchworm \\
$45^{\circ}$ & 1,5 & Inchworm \& Camera \\
$90^{\circ}$ & 1,3 & Inchworm \& Camera \\
$90^{\circ}$ & 1 & Inchworm, Rotation \& Camera \\
$0^{\circ}$ & 3 & Helicoidal v1.0 \\
$90^{\circ}$ & 1,2 & Helicoidal v1.0 \\
\hline
\end{tabular}
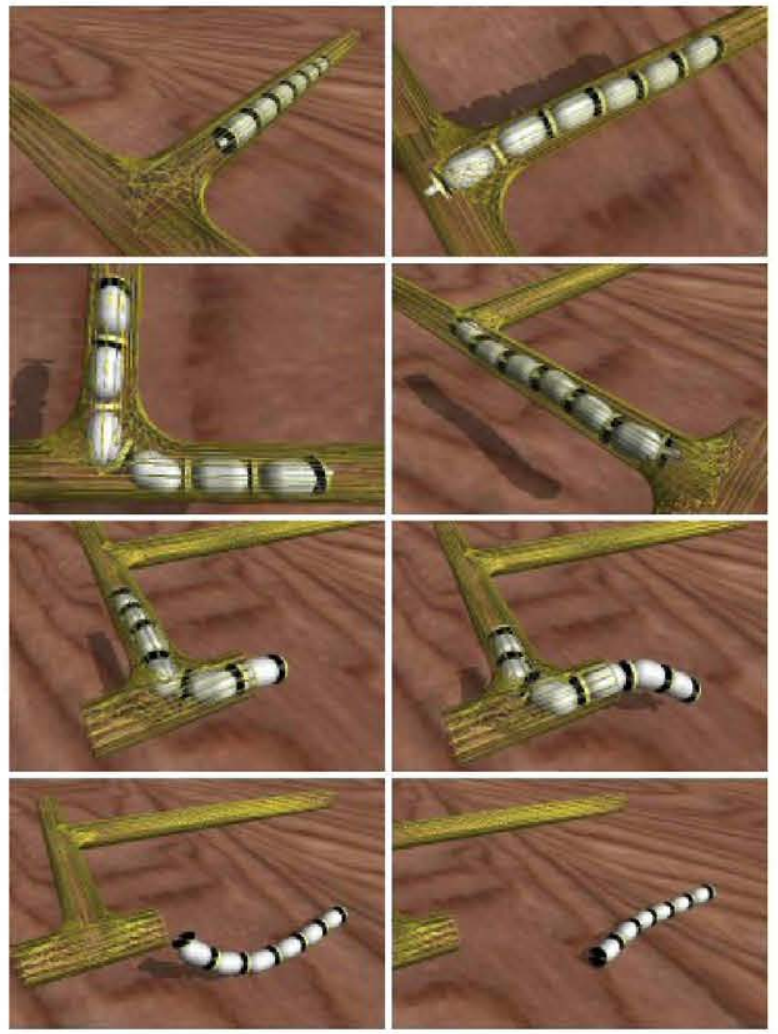

Fig. 18. Negotiating an elbow

\section{Conclusions}

Although there are many designs concerning homogeneous modular robots, there is not a single one that is truly heterogeneous. As we have seen, the heterogeneous designs have a single type of drive module and the other one is passive. 
In this paper a proposal for a multi-drive heterogeneous modular pipe-inspection microrobot has been presented. Both the electromechanical and the control scheme have been described. This architecture allows the control (based on behaviors) of different modules and the possibility of adding new ones without reconfiguring the system.

The prototype modules already developed and the ones that are in design process have been described and the simulation environment that is being used for testing has been presented. Some tests performed concerning the use of accelerometers have been shown.

Future work will be focused on the development of the real modules and testing the algorithms in the real robot.

\section{Acknowledgements}

The authors want to thank the support of the National Spanish Research Program (Project MEC DPI2005-08932-C02) and the "Consejeria de Educacion de la Comunidad de Madrid y el Fondo Social Europeo (F.S.E.)".

The authors would also like to thank the contributions of Laura Santos and the "Dpto. de Ing. Mecanica y Fabricacion", "Division de Ingenieria de Fabricacion" and "Taller de Prototipado Rapido". E.T.S.I.Industriales, UPM.

\section{References}

Arkin, R. C. [1998] Behavior-Based Robotics (MIT Press).

Brener, N., BenAmar, F. and Bidaud, P. [2004] Analysis of self-reconfigurable modular systems: a design proposal for multi-modes locomotion, in IEEE International Conference on Robotics and Automation, pp. 996-1001.

Brunete, A., Hernando, M. and Gambao, E. [2005] Modular multiconfigurable architecture for low diameter pipe inspection microrobots, in Proceedings of the 2005 IEEE International Conference on Robotics and Automation (ICRA) (Barcelona, Spain).

Brunete, A., Torres, J., Hernando, M. and Gambao, E. [2007] Multi-drive control for in-pipe snakelike heterogeneous modular micro-robots, in Proceedings of the 2007 IEEE International Conference on Robotics and Biomimetics (ROBIO).
Gonzalez, J., Zhang, H., Boemo, E. and Zhang, J. [2006] Locomotion of a modular robot with eight pitch-yaw-connecting modules, in 9th International Conference on Climbing and Walking Robots.

Huntsberger, T., Pirjanian, P., Trebi-Ollennu, A., Nayar, H. D., Aghazarian, H., Ganino, A. J. and Garrett, M. [2003] Campout: a control architecture for tightly coupled coordination of multirobot systems for planetary surface exploration, IEEE Transactions on Systems, Man and Cybernetics 33(5), 550-559.

Jantapremjit, P. and Austin, D. [2001] Design of a modular self-reconfigurable robot, in Australian Conference on Robotics and Automation.

Jorgensen, M., Ostergaard, E. and Lund, H. [2004] Modular atron: Modules for a self-reconfigurable robot, in Proceedings of the 2004 IEEE/RSJ International Conference on Intelligent Robots and Systems (Japan).

Klaassen, B. and Paap, K. [1999] Gmd-snake2: a snake-like robot driven by wheels and a method for motion control, in Proceedings of the 1999 IEEE International Conference on Robotics and Automation, pp. 3014-3019.

Kurokawa, H., Tomita, K., Kamimura, A., Yoshida, E., Kokaji, S. and Murata, S. [2005] Distributed self-reconfiguration control of modular robot m-tran, in IEEE International Conference on Mechatronics and Automation, pp. $254-259$.

Mataric, M. M. [1992] Behavior-based control: Main properties and implications, in Proceedings of the IEEE International Conference on Robotics and Autonomation, Workshop on Architectures for Intelligent Control Systems.

Pirjanian, P. [1999] Behaviour coordination mechanisms, Tech. rep., University of Southern California.

Roh, S. and Choi, H. [2004] Differential-drive in-pipe robot for moving inside urban gas pipelines, IEEE Transactions on Robotics .

Rus, D. and Vona, M. [2000] A physical implementation of the self-reconfiguring crystalline robot. in IEEE International Conference on Robotics and Automation, pp. 1726-1733.

Shen, W.-M., Krivokon, M., Chiu, H., Everist, J., Rubenstein, M. and Venkatesh, J. [2006] Multimode locomotion via superbot reconfigurable robots, Auton. Robots 20(2), 165-177.

Shen, W.-M., Lu, Y. and Will, P. [2000] Hormonebased control for self-reconfigurable robots, in Proceedings of the International Conference on Autonomous Agents (Barcelona, Spain). 
Suh, J., Homans, S. and Yim, M. [2002] Telecubes: mechanical design of a module for selfreconfigurable robotics, in IEEE International Conference on Robotics and Automation, pp. 4095-4101.

Unsal, C. and Khosla, P. K. [2000] Mechatronic design of a modular self-reconfigurable robotics system. in IEEE International Conference on Intelligent Robots and Systems, pp. 1742-1747.

Yim, M. [1994] New locomotion gaits. in Proceed- ings of the 1994 IEEE International Conference on Robotics and Automation, pp. 2508-2514.

Zhang, Y., Roufas, K. D. and Yim, M. [2001] Software architecture for modular self-reconfigurable robots, in Proceedings of the IEEE/RSJ Intl. Conf. on Intelligent Robots and Systems (IROS). Zykov, V., Mytilinaios, E., Adams, B. and Lipson, H. [2005] Self-reproducing machines, Nature 435, $163-164$. 